\title{
Aislamiento de espiroquetas intestinales Brachyspira spp., (enteropatógeno para el cerdo y otros animales domésticos) de muestras de heces remitidas a Hospital Público Isolation of intestinal spirochetes Brachyspira spp., (enteropathogenic to swine and other domestic animals) from stool samples submitted to public hospital
}

\author{
Enrique Corona Barrera, ${ }^{*}$ Diana I Salvador Trujillo, ${ }^{\ddagger}$ María G Ortiz Medina, ${ }^{\S}$ \\ Hugo Barrios García,* José Vázquez Villanueva,* Alexandra VanKley\| \\ * Facultad de Medicina Veterinaria y Zootecnia, Universidad Autónoma de Tamaulipas. \\ ‡ Hospital Civil de Ciudad Victoria, Tamaulipas «Dr. José Macías Hernández», México. \\ ${ }^{\S}$ Hospital Infantil de Tamaulipas. \\ " Science Department, Stephen Austin State University, Texas USA.
}

RESUMEN

Brachyspira spp. son espiroquetas intestinales y patógenos importantes en medicina veterinaria, el cerdo es el hospedador del que más estudios se han reportado. Las espiroquetas intestinales son responsables de infecciones en el tracto intestinal que se manifiestan como diarrea mucohemorrágica, colitis y trastornos intestinales en varios hospederos incluyendo al humano. Dos especies de Brachyspira afectan a las personas, Brachyspira pilosicoli (también afecta al cerdo y a otros animales domésticos) y Brachyspira aalborgi. El objetivo de este trabajo fue demostrar la presencia de espiroquetas intestinales del género Brachyspira en muestras de heces remitidas a laboratorio de parasitología de dos hospitales públicos de Ciudad Victoria, Tamaulipas. Se obtuvieron alícuotas de muestras de heces del Hospital Civil de Ciudad Victoria $(n=160)$ y del Hospital Infantil de Tamaulipas $(n=65)$. Las muestras fueron sembradas en medio de cultivo selectivo e incubadas en jarra en condiciones de anaerobiosis a $42{ }^{\circ} \mathrm{C}$ durante siete días para aislamiento de Brachyspira. De 225 muestras, cinco fueron positivas al aislamiento bacteriológico, confirmando la presencia de formas espiroquetales características Gram -vo por microscopia (100x). De primo-aislamientos o pases subsecuentes de los aislados se obtuvo ADN para la identificación molecular de los especímenes por medio de 16 S rDNA-PCR. La ocurrencia de aislamiento de espiroquetas intestinales fue de $2.2 \%$ (5/225), de las muestras positivas, cuatro fueron de muestras del Hospital Infantil de Tamaulipas y de esas, dos fueron de
ABSTRACT

Brachyspira spp. are intestinal spirochetes that are important in veterinary medicine, the pig is the host for which more studies have been published. The intestinal spirochetes are responsible intestinal infections that cause muco-hemorragic diarrhea, colitis and intestinal disorders in various hosts including humans. Two species of Brachyspira affect people, Brachyspira pilosicoli (also affects the pig and other domestic animals) and Brachyspira aalborgi. The aim of this work was to demonstrate evidence of the genus Brachyspira in fecal samples sent to parasitology laboratory of two public hospitals in Ciudad Victoria, Tamaulipas, Mexico. Fecal samples were obtained from Hospital Civil de Tamaulipas (Civil Hospital of Tamaulipas, $\mathrm{n}=160$ ) and from Hospital Infantil de Tamaulipas (Children Hospital of Tamaulipas, $n=65$ ). Samples were seeded onto selective bacteriological medium and incubated in anaerobic jar at $42{ }^{\circ} \mathrm{C}$ for 7 days for isolation of Brachyspira. Out of 225 samples, 5 were positive to bacteriological isolation, the spirochetal forms Gram -ve were confirmed by microscopy (100x). From primeisolates and subsequent passages DNA was extracted for the molecular identification of specimens by the 16S rDNAPCR. The occurrence of intestinal spirochetes isolation was 2.2\% (5/225), of the positive samples, 4 were from Children Hospital of Tamaulipas and out of those, 2 from children of 9 and 14 months old, and the other 2 from children of 9 and 14 years of age. The results of this work showed evidence of intestinal spirochetes in fecal samples sent to the parasitology lab of the public hospitals Financiamiento: Este trabajo fue realizado con financiamiento de una beca del Programa para el Desarrollo del Profesional
Docente de la SEP, PRODEP-UAT-PTC-201.

Conflicto de intereses: Ninguno.

https://dx.doi.org/10.35366/95648

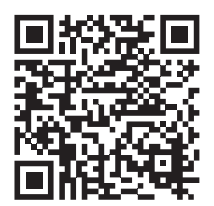


niños de nueve y 14 meses de edad, y las otras dos de niños de nueve y 14 años de edad. Los resultados muestran la presencia de Brachyspira en muestras de heces remitidas a los hospitales públicos incluidos en la investigación. Un dato relevante en este trabajo fue el aislamiento de espiroquetas intestinales de muestras de heces de niños, lo cual deja una interrogante en el mecanismo de transmisión. Este trabajo representa el primer reporte de presencia de este enteropatógeno en el sector salud en México.

Palabras clave: Espiroquetas intestinales, Brachyspira, enteropatógeno, zoonosis.

\section{INTRODUCCIÓN}

Las espiroquetas intestinales (EI) son bacterias anaeróbicas tolerantes al oxígeno, lo cual las coloca en la categoría de fastidiosas para trabajar en el laboratorio. En tiempos recientes varias especies de El han sido reconocidas, algunas de las cuales han sido reportadas en el cerdo; sin embargo, hay otros hospederos como aves (gallinas de postura) para los cuales estas bacterias también son patógenas, dentro de esos hospederos se incluye al humano.

Debido a los cambios en la taxonomía de las El, en la actualidad la familia Brachyspiracea incluye al género Brachyspira, ${ }^{1}$ que incluye a un amplio número de especies de las cuales $B$. innocens es considerada apatógena y se encuentra en el tracto intestinal del cerdo. Otras especies de Brachyspira en medicina veterinaria por su patogenicidad son particularmente importantes en el cerdo, Brachyspira hyodysenteriae (antes Treponema hyodysenteriae y/o Serpulina hyodysenteriae), ${ }^{2,3}$ Brachyspira pilosicoli, ${ }^{4}$ Brachyspira intermedia y Brachyspira murdochii. ${ }^{5}$ Otras especies de Brachyspira se encuentran otros hospederos como aves, Brachyspira alvinipulli ${ }^{6}$ y Brachyspira suanatina ${ }^{7}$ o el perro, Brachyspira canis, ${ }^{8}$ y recientemente otra especie patógena se encontró en el cerdo, Brachyspira hampsonii. ${ }^{9}$

En el cerdo, B. pilosicoli es responsable de una condición patológica denominada espiroquetosis colónica porcina, ${ }^{4}$ la cual se caracteriza por una diarrea catarral (exceso de moco en heces) que afecta el desarrollo normal de los cerdos. En humanos, $B$. pilosicoli es responsable de una condición patológica del colon denominada espiroquetosis colónica. Por su parte, $B$. aalborgi está asociada a la patología conocida como espiroquetosis intestinal humana. ${ }^{10}$ Varios reportes han dejado evidencia que la espiroquetosis intestinal en humanos es una condición intestinal que genera dolor abdominal, diarrea recurrente, colitis y pérdida de peso, ya que included in this research. An important fact was the isolation of intestinal spirochetes from fecal samples of children, for which the mechanism of transmission remains uncertain. This work is the first report on the presence of the enteropathogen Brachyspira in the public health sector in Mexico.

Keywords: Intestinal spirochetes, Brachyspira, enteropathogen, zoonosis.

se han documentado casos en varios países, por ejemplo, Dinamarca, ${ }^{10}$ Italia, ${ }^{11}$ Estados Unidos, ${ }^{12}$ Noruega ${ }^{13}$ y Australia, ${ }^{14}$ entre otros. En México no hay reportes consistentes del aislamiento de El de humanos a nivel de muestras de hospital; sin embargo, existe reporte de su aislamiento en muestras colectadas de comunidades rurales junto con muestras de animales domésticos en las que se mostró la evidencia de su presencia en muestras de animales domésticos y de humanos. ${ }^{15}$

En el estado de Tamaulipas no existen reportes de la ocurrencia de Brachyspira spp. asociados a trastornos intestinales en humanos, por lo que el objetivo de este trabajo fue determinar la presencia de El en muestras de heces referidas para estudios coproparasitoscópicos en hospital público en Ciudad Victoria, Tamaulipas, y lograr la identificación molecular de los aislados correspondientes.

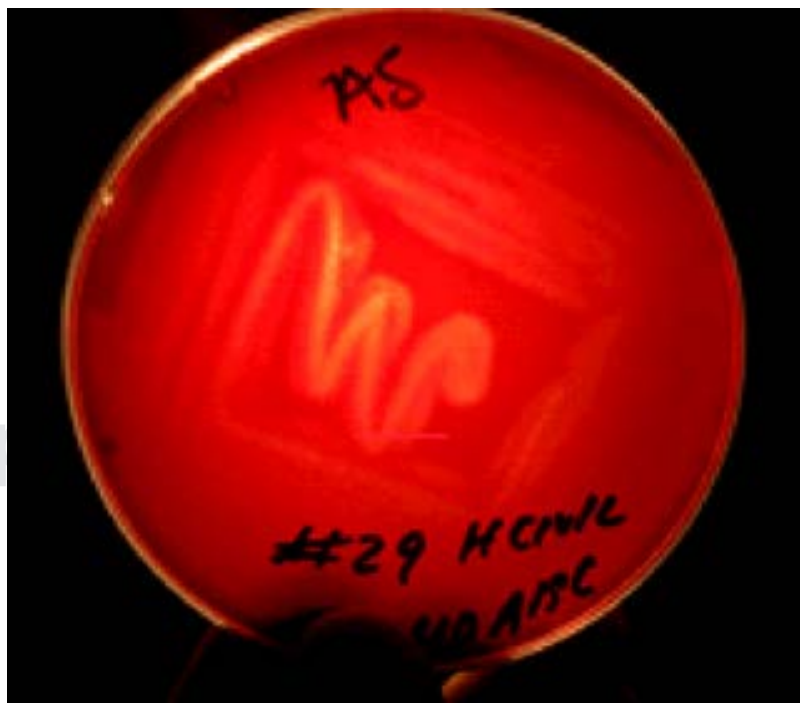

Figura 1: Aislamiento bacteriológico de El en Brachyspira Selective Medium-BSM, se observa hemólisis y crecimiento característico en avanzada de Brachyspira spp. 


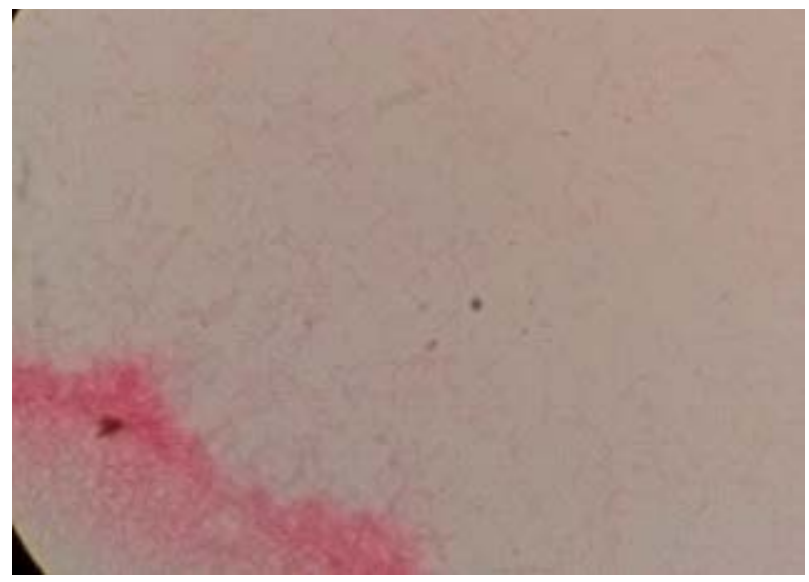

Figura 2: Formas espiroquetales de Brachyspira, Gram negativas observadas al microscopio óptico con objetivo de inmersión (100x).

\section{MATERIAL Y MÉTODOS}

Esta investigación se realizó en colaboración con investigadores de dos hospitales públicos del estado de Tamaulipas: el Hospital Civil de Ciudad Victoria (HCCV) y el Hospital Infantil de Tamaulipas (HIT). Durante el transcurso de este estudio de tipo observacional no se tuvo contacto con las personas (pacientes) que remitieron muestras de heces al laboratorio de los hospitales para detección de parásitos gastrointestinales por medio de estudios coproparasitoscópicos.

Para realizar el trabajo de laboratorio sólo se obtuvo una porción mínima (alícuota, menor a $1 \mathrm{~g}$ ) de heces de la muestra original, la cual se transfirió a un microtubo tipo Eppendorf $(1.5 \mathrm{~mL})$ y se transportaron en caja de unicel (hielera) con refrigerantes para preservarlas a baja temperatura hasta llegar al laboratorio de Microbiología de la Facultad de Medicina Veterinaria y Zootecnia de la Universidad Autónoma de Tamaulipas, donde se procesaron por bacteriología, habiendo transcurrido no más de 24 horas de haber sido colectadas. Se obtuvieron alícuotas de un total de 225 muestras de heces, del HCCV ( $\mathrm{n}=$ $160)$ y del HIT $(n=65)$. Normalmente, el esquema para remitir muestras al laboratorio para exámenes coproparasitoscópicos es en serie de tres, por lo que de algunas muestras se obtuvo la serie de tres y de otras sólo una o dos muestras de la serie de tres.

Las muestras se sembraron en medio del cultivo selectivo para Brachyspira, BSM (Brachyspira Selective Medium)-Agar Columbia no. 2 (Oxoid $^{\circledR}$ UK), suplementado con $8.0 \%$ de sangre de caballo y un compuesto de espectinomicina $500 \mathrm{mg} / \mathrm{L}$, vancomicina $25 \mathrm{mg} / \mathrm{L}$ y colistina $25 \mathrm{mg} / \mathrm{L}$. Los sembrados fueron incubados por siete días a $42^{\circ} \mathrm{C}$, en jarra de anaerobiosis usando el sistema GasPak (AnaeroGen Oxoid ${ }^{\circledR}$, UK). Después de la incubación los sembrados fueron revisados buscando observar primo-aislamiento con el crecimiento característico de Brachyspira spp., (hemólisis y crecimiento en avanzada). Para confirmar la presencia de El en primo-aislamientos se realizaron preparaciones de frotis, se tiñeron con tinción de Gram y observaron al microscopio (Carl Zeiss Primo Star, Germany) con objetivo de inmersión (100x), para detectar las formas espiroquetales características de Brachyspira. De primo-aislamientos de El se realizaron pases subsecuentes hasta obtenerlas en cultivo puro.

A partir de aislamientos se realizó una extracción de ADN (QIAamp PowerFeca ${ }^{\circledR}$ DNA Kit, QIAGEN Germany) para después someterlo a una reacción en cadena de la polimerasa (PCR) flanqueando el gen 16S rDNA y usando los iniciadores Brachys piloaalbor F 5' (5'-GCGAACTGGTGAGTAACACG-3') y Brachys pilo-aalbor R 3' (5'-GAGTCTGGGCCGTATCTCAG-3') en reacciones de $50 \mu \mathrm{L}$ volumen final, a una concentración de $0.8 \mu \mathrm{mol} / \mathrm{L}$, mediante las condiciones de termociclado: $94^{\circ} \mathrm{C}$ por 1 minuto, 58 ${ }^{\circ} \mathrm{C}$ p or 1 minuto y $72{ }^{\circ} \mathrm{C}$ por un minuto por 40 ciclos, generando un producto de $241 \mathrm{pb}$, de acuerdo con la metodología descrita por..$^{12}$ Para la reacción de PCR se incluyó la cepa ATCC-51139 de B. pilosicoli como control positivo. Los amplicones fueron movilizados por electroforesis en geles de agarosa al $1.5 \%$, teñidos con bromuro de etidio y visualizados en fotodocumentador (Gel Doc®, BioRad USA).

\section{RESULTADOS}

De 225 muestras procesadas para el aislamiento bacteriológico de Brachyspira, cinco fueron positivas al detectarse el crecimiento característico de El en cultivo (crecimiento hemolítico y en avanzada) (Figura 1), en las que la presencia de formas espiroquetales Gram negativas fue confirmada por microscopia óptica con objetivo de inmersión (100x). Una vez confirmada la pureza de los pases subsecuentes en preparaciones microscópicas se consideraron como aislados de Brachyspira spp., de los cuales se obtuvo evidencia fotográfica (Figura 2).

La ocurrencia de aislamiento fue de 2.2\% (5/225), de los cuales cuatro muestras fueron del HIT, dando una ocurrencia de $6.1 \%(4 / 65)$, de las cuales dos 
fueron de niños de nueve y 14 meses de edad y las otras dos muestras positivas fueron de pacientes de nueve y 14 años de edad. La muestra positiva a aislamiento proveniente del HCCV fue de un individuo femenino de 50 años de edad. Del ADN extraído de los aislados de El se confirmó la identidad de Brachyspira a través del 16S rDNA-PCR, observando el amplicón característico de 241 pb en gel de agarosa teñido con Bromuro de Etidio y visualizado en transiluminador (Figura 3).

\section{DISCUSIÓN}

Datos de la situación epidemiológica de El van de acuerdo a la metodología diagnóstica usada para la identificación, sea en biopsia de tejido intestinal (colon), aislamiento bacteriológico y/o pruebas moleculares (PCR). Las El son consideradas como fastidiosas para trabajar en el laboratorio, ${ }^{6}$ por lo que varios reportes han basado los datos de prevalencia en detección molecular; sin embargo, lograr aislamiento es una evidencia contundente para demostrar la presencia del agente en la muestra.

En este estudio se obtuvo una ocurrencia de aislamiento de El de 2.2\% (5/225) de muestras de heces,

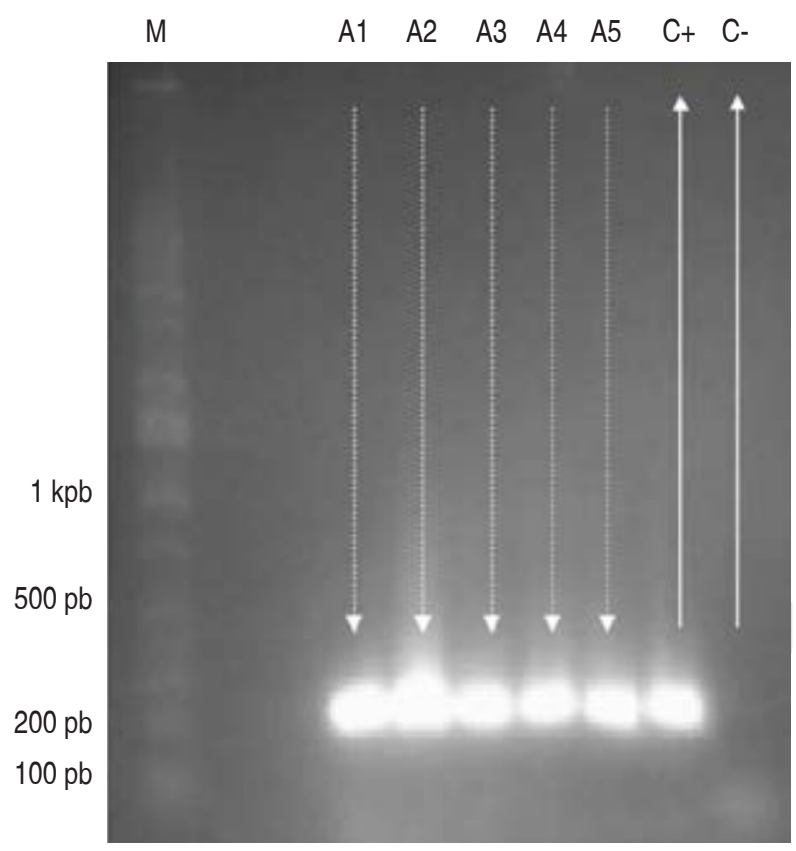

Figura 3: Amplicón de 241 pb producto de Brachyspira 16S rDNAPCR en gel de agarosa + Bromuro de Etidio. A1-A5 aislados de EI de muestras de heces de hospital público; C+ Control positivo ATCC51139 B. pilosicoli y C-control negativo sin templete de ADN. que fue confirmada por 16S rDNA-PCR, lo cual dejó claro que se trata de aislados de Brachyspira. En otros estudios han reportado prevalencia de 2.5 a $16.0 \%$ en individuos de países desarrollados. ${ }^{10,13}$ No obstante, en países en desarrollo se reporta una prevalencia más alta (33.0\%) de acuerdo con datos de los últimos 20 años, es importante el hecho que las El se han encontrado tanto en individuos con diarrea y en aquellos sin diarrea, siendo mayor la prevalencia en individuos con trastornos intestinales. ${ }^{16,17}$ Para el presente estudio, las muestras fueron remitidas a hospital público para estudios coproparasitoscópicos y no necesariamente para estudio de aislamiento de Brachyspira spp.

En un estudio previo en México realizado en comunidades rurales de Irapuato, Guanajuato, se obtuvo una ocurrencia de primo-aislamiento de EI de cuatro hospederos de esas comunidades, cerdo, perro, aves (gallinas) y humano, en la que para las muestras de humano se encontró una ocurrencia de $16.7 \%(22 / 131)$ y en general una ocurrencia de $18.1 \%(86 / 473)$ del total de muestras de los cuatro hospederos, los aislados de El de las heces de humano de las comunidades rurales muestreadas en 2014 fueron compatibles con B. pilosicoli por 23S rDNA RFLP-PCR. ${ }^{15}$

Dado que $B$. pilosicoli es capaz de colonizar a varios hospederos, el haberla encontrado en sus hospederos en comunidades rurales toma relevancia como un problema de salud pública. Estudios realizados en Australia en niños de comunidades de aborígenes en el área de Kimberly y Darwin, encontraron que $32.0 \%$ (59/181) de las muestras fueron positivas para El; sin embargo, los datos de ocurrencia de El para habitantes urbanos de ciudades del Noroeste de Australia fue de $1.2 \%$ (8/695). En ese estudio se reportó que la edad de las personas era entre dos y 18 años de edad, siendo la ocurrencia de El mayor en niños menores a dos años de edad y que algunos de los niños fueron muestreados consecutivamente durante tres años, obteniéndose en algunos casos aislamiento positivo en la primera y la segunda colecta de muestra, y en otros casos en la primera y la tercera colecta de muestra. ${ }^{17}$ En el presente estudio se detectó la presencia de El en muestras de niños entre nueve meses y 14 años de edad y en algunos casos también se detectó la presencia de EI en muestras seriadas, por ejemplo, de las muestras seriadas de tres se consiguió detección de todas 0 en otros casos en al menos una de las tres muestras, lo cual coincide en parte con lo reportado en el 
Rev Latin Infect Pediatr 2020; 33 (3): 125-130

trabajo de Lee y Hampson, en 1992, en aborígenes australianos. ${ }^{17}$

Son dos las especies de Brachyspira que afectan al humano: $B$. aalborgi y $B$. pilosicoli; estudios de muestreo de heces y PCR en India encontraron una prevalencia de $6.0 \%(19 / 316)$ para $B$. aalborgi y de $25.6 \%(80 / 316)$ para $B$. pilosicoliy una prevalencia de $3.2 \%$ para la presencia de ambas El. ${ }^{18}$ En ese estudio también se determinaron algunos factores de riesgo para la ocurrencia de Brachyspira en los habitantes de esos lugares, identificándose el radicar en Balipara, que otros miembros de la familia estén colonizados y tomar agua de pozo asociados $(p<0.05)$ con la presencia de El; es interesante el factor de riesgo que otros miembros de la familia estén colonizados.

En otro estudio realizado en Indonesia también se encontró como factor de riesgo para la ocurrencia de $B$. pilosicoli $(p<0.01)$ el que otros miembros de la familia tengan trastornos intestinales y tomar agua de pozo, además de niños de 2-5 años de edad e identificación de protozoarios. ${ }^{19}$ Lo anterior podría explicar en parte lo encontrado en el presente trabajo, ya que se aislaron El de muestras de heces de niños menores de un año porque a esa edad los niños normalmente dependen del cuidado de los padres, principalmente de la madre; por lo que el mecanismo de transmisión podría ser el contacto estrecho que se da entre hijos y padres, estos últimos podrían ser la fuente de contagio.

Sin duda, la epidemiología de Brachyspira spp. es compleja porque es un patógeno intestinal que afecta a varios hospederos animales causando diarrea, colitis hemorrágica (disentería porcina) o colitis catarral y/o trastornos intestinales, por lo que su estudio merece atención por ser un problema de salud pública desde la perspectiva de las zoonosis.

En este trabajo se demostró la presencia de EI en muestras de heces remitidas a dos hospitales públicos de Ciudad Victoria, Tamaulipas. Brachyspira se aisló de muestras de un paciente adulto y de varios niños, siendo mayor la frecuencia de aislamiento de niños y la identidad molecular de los aislados fue realizada por $16 \mathrm{~S}$ rDNA-PCR, con lo que quedó evidenciado que Brachyspira tiene presencia en habitantes en el estado de Tamaulipas. Además, un dato relevante en este trabajo fue el aislamiento de Brachyspira de muestras de heces de niños de muy corta edad (nueve y 14 meses), lo cual deja una interrogante en el mecanismo de transmisión. Este trabajo representa el primer reporte de presencia de Brachyspira spp. en el sector salud en México.

\section{REFERENCIAS}

1. Ochiai S, Adachi Y, Mori K. Unification of the genera Serpulina and Brachyspira and proposals of Brachyspira hyodysenteriae comb. nov., Brachyspira innocens comb. nov. and Brahcypira pilosicoli comb. nov. Microbiol Immunol. 1997; 41 (6): 445-452.

2. Taylor DJ, Alexander TJL. The production of dysentery in swine by feeding cultures containing a spirochaete. Br Vet $\mathrm{J}$. 1971; 127: 58-61.

3. Stanton TB. Proposal to change the genus designation Serpula to Serpulina gen.nov. containing the species Serpulina hyodysenteriae comb.nov. and Serpulina innocens comb. Nov. Int J Syst Bacteriol. 1992; 42: 189-190.

4. Trott DJ, Stanton TB, Jensen NS, Duhamel GE, Johonson JL, Hampson DJ. Serpulina pilosicoli sp. nov.: The agent of porcine intestinal spirochetosis. Int J Syst Bacteriol. 1996; 46: 206-215.

5. Hampson DJ, La T. Reclassification of Serpulina intermedia and Serpulina murdochii in the genus Brachyspira as Brachyspira intermedia comb. nov. and Brachyspira murdochii comb. nov. Int J Syst Evol Microbiol. 2006; 59: 1009-1012.

6. Stanton TB. Physiology of ruminal and intestinal spirochaetes. En: Hampson DJ, Stanton TB (Eds.) Intestinal Spirochaetes of Domestic Animals and Humans. New York, USA. CAB International. 1997, pp 7-45.

7. Rasbäck T, Jansson DS, Johansson KE, Fellström C. A novel enteropathogenic, strongly haemolytic spirochaete isolated from pig and mallard, provisionally designated 'Brachyspira suanatina' sp. nov. Environ Microbiol. 2007; 9: 983-991.

8. Duhamel GE, Trott DJ, Muniappa N, Mathiesen MR, Tarasiuk K, Lee JI, Hampson DJ. Canine intestinal spirochetes consist of Serpulina pilosicoli and a newly identified group provisionally designated "Sepulina canis" sp. nov. J Clin Microbiol. 1998; 36 (8): 2264-2270.

9. Harding JCS, Chirino-Trejo M, Fernando C, Jacobson M, Forster Z, Hill JE. Detection of a novel Brachyspira species associated with haemorrhagic and necrotizing colitis. Proceedings of the Int Pig Vet Soc Congr., Vancouver, Canada: IPVS. 2010, p. 740.

10. Hovind-Hougen K, Birch-Andersen A, Henrik-Nielsen R, Orholm M, Pedersen JO, Teglbjaerg PS, et al. Intestinal spirochetosis: morphological characterization and cultivation of the spirochete Brachyspira aalborgi gen. nov., sp. nov. J Clin Microbiol. 1982; 16(6): 1127-1136.

11. Calderaro A, Gorrini C, Peruzzi S, Piccolo G, Dettori G, Chezzi C. Occurrence of human intestinal spirochetosis in comparison with infections by other enteropathogenic agents in an area of the Northern Italy. Diagn Microbiol Infect Dis. 2007; 59: 157-163.

12. Koteish A, Kannangai R, Abraham SC, Torbenson M. Colonic spirochetosis in children and adults. Am J Clin Pathol. 2003; 120: 828-832.

13. Lindboe CF. The prevalence of human intestinal spirochetosis in Norway. Anim Health Res Rev. 2001; 2: 117-119.

14. Brooke CJ, Riley TV, Hampson DJ. Comparison of prevalence and risk factors for faecal carriage of the intestinal spirochaetes Brachyspira aalborgi and Brachyspira pilosicoli in four Australian populations. Epidemiol Infect. 2006; 134 (3): 627-634.

15. Corona-Barrera E, González J, Navarrete A, López E, Toledo B, Hernández M, Ángel C, Gutiérrez A, Valencia 
Rev Latin Infect Pediatr 2020; 33 (3): 125-130

M, VanKley A. Aislamiento de espiroquetas intestinales del género Brachyspira de habitantes humanos de comunidades rurales de Irapuato, Gto. XV Reunión de Ciencias Médicas., León, Gto., Apr 22-24, 2015.

16. Barret SP. Intestinal spirochaetes in a Gulf Arab population. Epidemiol Infect. 1990; 164: 261-266.

17. Lee JI, Hampson DJ. Intestinal spirochaetes colonizing aborigines from communities in the remote north of Western Australia. Epidemiol Infect. 1992; 109 (1): 133-141.

18. Munshi MA, Traub RJ, Robertson ID, Mikosa ASJ, Hampson DJ. Colonization and risk factors for Brachyspira aalborgi and Brachyspira pilosicoli in humans and dogs on tea estates in Assam, India. Epidemiol Infect. 2003; 132: 137-144.
19. Margawani KR, Robertson ID, Brooke CJ, Hampson DJ Prevalence, risk factors and molecular epidemiology of Brachyspira pilosicoli in humans on the island of Bali, Indonesia. J Med Microbiol. 2004; 53 (Pt 4): 325-332.

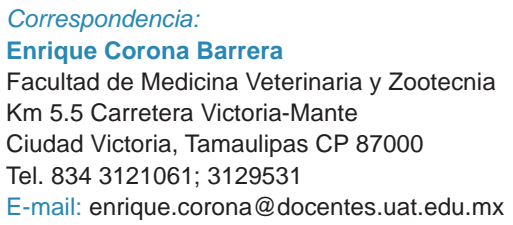

